\title{
ESTIMATING EXPOSURE RESPONSE FUNCTIONS USING AMBIENT POLLUTION CONCENTRATIONS
}

\author{
By Gavin Shaddick ${ }^{1 *}$ Duncan LeE ${ }^{2}$ James V. ZideK ${ }^{3}$ and Ruth \\ SALWAY $^{1}$, \\ University of Bath ${ }^{1}$ and University of Glasgow ${ }^{2}$ and University of British \\ Columbia $^{3}$
}

\section{SUPPLEMENTARY MATERIAL. \\ Contents:}

1. Using the pCNEM exposure simulator.

2. Modelling the effects of multiple lags.

3. Data for London, 1997, used in the paper.

1. Using the pCNEM exposure simulator. The pCNEM exposure simulator used in this paper is available for use via a WWW interface. The interface can be used to configure a model and to launch simulations. The site contains an existing model for $\mathrm{PM}_{10}$ exposures in the Vancouver area which is accessible to all users as a template.

The computer model consists of several components:

- the location,

- the demographic groups,

- the microenvironments,

- the sources,

- the model data, and

- the simulator.

The model data component is used to supply the ambient field and possibly the meteorological data corresponding to the area and the period for which the simulation will be done. The location component is used to define the mapping from individual's locations to the microenvironments defined in the simulation. The simulator component is used to launch simulations. The demographic groups component is used to edit and inspect the demographic groups defined for the model. The microenvironments component is used to edit and inspect the microenvironments defined for the model. The sources

\footnotetext{
*Email address for corresponding author - g.shaddick@bath.ac.uk
} 
component is used to edit and inspect the sources defined for the model.

Prospective users of the pCNEM system should apply to jim@stat.ubc.ca.

Once registered, users can

- upload their own databases;

- add their own 'micro-environments (MEs)', key building blocks for the model;

- add sources interior to each ME;

- fit appropriate source parameters

- make replicate runs of the model remotely

- download outputs in spreadsheet format to their own PC for further analysis and application.

More detail is available on-line by accessing the 'help' screens.

2. Modelling the effects of multiple lags. For simplicity model (3.6) and the discussion of ecological bias was based on only including a single lagged value of exposure in the model, which might commonly be chosen to be one or two (Dominici et al. (2000)). However the latency over which the health effects manifest themselves is unknown, and so the choice of a single lag can be problematic. Furthermore pollution concentrations on successive days are likely to be highly correlated, meaning that the observed association at the chosen lag may be an artifact of a true association at a different lag. One solution might be to include multiple lags $\mathbf{X}_{t}=\left(X_{t}, X_{t-1}, \ldots, X_{t-L}\right)$ in the model, with a corresponding vector of effects, $\gamma=\left(\gamma_{0}, \ldots, \gamma_{L}\right)$. To allow for ecological bias in this situation would require the addition of covariance terms to the mean function. For example, considering the common simplification that the exposure response function $g$ is linear, i.e. $g(x)=x$, and that the daily exposure distribution is normal, i.e. $\mathbf{X}_{t} \sim \mathrm{N}\left(\tilde{\boldsymbol{\lambda}}_{t}^{(1)}, \tilde{\boldsymbol{\lambda}}_{t}^{(2)}\right)$, then the mean function becomes

$$
\mu_{t}=\exp \left(\mathbf{z}_{t}^{\mathrm{T}} \boldsymbol{\alpha}\right) \exp \left(\boldsymbol{\gamma}^{\mathrm{T}} \tilde{\boldsymbol{\lambda}}_{t}^{(1)}+\boldsymbol{\gamma}^{\mathrm{T}} \tilde{\boldsymbol{\lambda}}_{t}^{(2)} \boldsymbol{\gamma} / 2\right)
$$

where $\tilde{\boldsymbol{\lambda}}_{t}^{(2)}$ is an $L+1$ dimensional covariance matrix. Adopting a more realistic multivariate log-normal exposure model yields an approximate mean function 


$$
\begin{aligned}
\mu_{t} & =\exp \left(\mathbf{z}_{t}^{\mathrm{T}} \boldsymbol{\alpha}\right) \exp \left(\boldsymbol{\gamma}^{\mathrm{T}} \tilde{\boldsymbol{\lambda}}_{t}^{(1)}+\gamma^{\mathrm{T}} \tilde{\boldsymbol{\lambda}}_{t}^{(2)} \gamma / 2+\tilde{\boldsymbol{\lambda}}_{t}^{(3)}\right) \\
\tilde{\boldsymbol{\lambda}}_{t}^{(3)} & =\frac{1}{6} \sum_{k=0}^{L} \gamma_{k}^{3} C_{k}^{3}+\frac{1}{2} \sum_{k \neq j} \gamma_{k}^{2} \gamma_{j} C_{k j}^{2,1}+\sum_{k \neq j \neq i} \gamma_{k} \gamma_{j} \gamma^{i} C_{k j i}^{1,1,1},
\end{aligned}
$$

which is the natural multivariate extension to the mean function in model (3.6). Here $\tilde{\boldsymbol{\lambda}}_{t}^{(3)}$ denote the collection of third central moments, namely: (i) $C_{k}^{3}=\mathbb{E}\left[\left(X_{t-k}-\lambda_{t-k}^{(1)}\right)^{3}\right]$; (ii) $C_{k j}^{21}=\mathbb{E}\left[\left(X_{t-k}-\lambda_{t-k}^{(1)}\right)^{2}\left(X_{t-j}-\lambda_{t-j}^{(1)}\right)\right]$; and (iii) $C_{k j i}^{111}=\mathbb{E}\left[\left(X_{t-k}-\lambda_{t-k}^{(1)}\right)\left(X_{t-j}-\lambda_{t-j}^{(1)}\right)\left(X_{t-i}-\lambda_{t-i}^{(1)}\right)\right]$. This mean function can replace the one in equation (3.6), with the cross terms of second and third order being fixed prior to estimation. However for computational efficiency we remove the cross terms of the third central moment (namely $\left(C_{k j}^{21}, C_{k j i}^{111}\right)$ ) when fitting this model.

This mean function is likely to be unsatisfactory however, due to the high correlation amongst the lagged exposures, meaning that the results will suffer from collinearity problems reducing the accuracy of the effect estimates and inflating the credible intervals. One solution is to use a distributed lag model (DLM, see Zanobetti et al. (2000) and Welty et al. (2005)), which includes multiple lags in the model but reduces the effects of collinearity by putting constraints on the coefficients. An alternative to multiple lag models is Bayesian model averaging, which has been applied in an air pollution context (Clyde (2000)) to estimate the average (over different models) association over a number of lags. Here we adopt the DLM approach of Zanobetti et al. (2000), by constraining the coefficients using a Bayesian penalised spline (Lang and Brezger (2004)), with a variance term controlling the amount of smoothing across the lags. Therefore the distributed lag component of our model has the form

$$
\begin{aligned}
\mu_{t} & =\exp \left(\mathbf{z}_{t}^{\mathrm{T}} \boldsymbol{\alpha}\right) \exp \left(\boldsymbol{\gamma}^{\mathrm{T}} \tilde{\boldsymbol{\lambda}}_{t}^{(1)}+\boldsymbol{\gamma}^{\mathrm{T}} \tilde{\boldsymbol{\lambda}}_{t}^{(2)} \boldsymbol{\gamma} / 2+\tilde{\boldsymbol{\lambda}}_{t}^{(3)}\right) \\
\gamma_{j} & =S(j \mid \boldsymbol{\beta}, L+1) \quad \text { for } j=0, \ldots, L \\
\beta_{i} & \sim \mathrm{N}\left(\beta_{i-1}, \psi\right) \text { for } j=2, \ldots, L+1
\end{aligned}
$$

where $S(j \mid \boldsymbol{\beta}, L+1)$ is a natural cubic spline of lag number with $L+1$ degrees of freedom and parameter vector $\boldsymbol{\beta}=\left(\beta_{1}, \ldots, \beta_{L+1}\right)$. Therefore in the model above the collection of air pollution effects $\gamma=\left(\gamma_{0}, \ldots, \gamma_{L}\right)$ are modelled by a natural cubic spline of lag number with $L+1$ basis functions. This gives the spline as many degrees of freedom as there are effect parameters $\gamma_{j}$, meaning that without any constraints it would enforce no smoothing 
across the individual $\gamma_{j}$. In this case the model is essentially the same as a multiple lag model, and will suffer from collinearity problems. Therefore to constrain the exposure effect estimates $\gamma_{j}$, we penalise the coefficients of the spline $\boldsymbol{\beta}$ by forcing them to follow a random walk prior as suggested by Lang and Brezger (2004). The strength of this prior for $\boldsymbol{\beta}$ is controlled by a variance component $\psi$, which acts as a smoothing parameter in this model. If $\psi$ is large then the random walk prior has little impact on $\boldsymbol{\beta}$, and the effect estimates $\gamma_{j}$ are effectively unconstrained (as in a multiple lag model), meaning that collinearity problems are likely to persist. In contrast as $\psi \rightarrow 0$ the random walk prior becomes stronger, which forces the elements of $\boldsymbol{\beta}$ and hence the effect estimates $\boldsymbol{\gamma}$ to become more similar to each other. In the limit of a zero variance the effect estimates $\left(\gamma_{0}, \ldots, \gamma_{L}\right)$ are all identical, meaning that only a single parameter is being estimated which removes the problem of collinearity.

3. Data for London, 1997, used in the paper. There are two comma delimited files which contain the data used in this paper;

(i) dataset.csv - contains mortality, weather and ambient pollution data (by day).

(ii) personalexposures.csv - 800 (simulated) personal exposures (for each day).

\section{References.}

Clyde, M. (2000). Model uncertainty and health effect studies for particulate matter. Environmetrics 6, 745-763.

Dominici, F., J. Samet, and S. Zeger (2000). Combining evidence on air pollution and daily mortality from the 20 largest US cities: a hierarchical modelling strategy. Journal of the Royal Statistical Society Series A 163, 263-302.

Lang, S. and A. Brezger (2004). Bayesian P-Splines. Journal of Computational and Graphical Statistics 13, 183-212.

Welty, L., S. Zeger, and F. Dominici (2005). Bayesian distributed lag models: Estimating effects of particulate matter air pollution on daily mortality. Environmental Health Perspectives 96, 1-32.

Zanobetti, A., M. Wand, J. Schwartz, and L. Ryan (2000). Generalized additive distributed lag models: quantifying mortality displacement. Biostatistics 1, 279-292. 